\title{
Clinical research of insulin glargine $U 300$ in type 1 diabetes mellitus patients with frequent hypoglycaemia: real-world experience
}

\author{
Savaş Volkan Kişioğluํㄹ Ahmet Suat Demir², Damla Tufekci², Yasemin Emur Gunay², Hulya Coskun², \\ Ozge Ucuncu², Irfan Nuhoglu², Mustafa Kocak², Serdar Karakullukcu², Halil Onder Ersoz ${ }^{2}$
}

\begin{abstract}
${ }^{1}$ Health Sciences University Kanuni Training and Research Hospital, Trabzon, Turkey ${ }^{2}$ Department of Endocrinology, Karadeniz Technical University, Trabzon, Turkey
\end{abstract}

Submitted: 2 February 2021

Accepted: 27 March 2021

Arch Med Sci Atheroscler Dis 2021; 6: e102-e108 DOI: https://doi.org/10.5114/amsad.2021.105562 Copyright (c) 2021 Termedia \& Banach

\section{Abstract}

Introduction: We aimed to see whether insulin glargine U300 can provide better blood glucose control while reducing hypoglycaemia in a more homogeneous population compared to previous studies.

Material and methods: The retrospective study included type 1 diabetes mellitus (T1DM) patients with frequent hypoglycaemia. For evaluation of fasting blood glucose, haemoglobin glycated $\left(\mathrm{HbA}_{1 c}\right)$ and weight at 6 months and 12 months (final), observation windows of 120-240 days (4-8 months) and 240-480 days (9-16 months) after insulin glargine U300 initiation, respectively, were permitted. Mean follow-up time was 12 months. Hypoglycaemia was defined as blood glucose level $<70 \mathrm{mg} / \mathrm{dl}$, either symptomatic or asymptomatic, measured in hospital or at home.

Results: Forty-four patients were included in the study, and 35 patients completed the study - 20 (57.1\%) females and 15 (42.9\%) males, with a mean age of $24.1 \pm 6.6$ years. Mean body mass index was $24.4 \pm 7.4 \mathrm{~kg} / \mathrm{m}^{2}$. A significant decrease was not found between baseline and $\mathrm{HbA}_{1 \mathrm{c}}$ values at 6 months ( $p=0.199$ ), but a significant decrease was found in the final period (between 9-16 months) $(p=0.025)$. Hypoglycaemic events occurred in all patients $(100 \%)$ before using insulin glargine U 300 , while the incidence of hypoglycaemic events gradually decreased to $74.3 \%, 68.6 \%$, and $68.6 \%$ between months $1-3,3-6$, and $6-9$, respectively. Of the 26 patients who declared their level of satisfaction, $23(88.5 \%)$ were satisfied, $2(7.7 \%)$ indicated that there was no significant difference, and $1(3.8 \%)$ patient was unsatisfied.

Conclusions: Over 9-16 months of follow-up, insulin glargine U300 led to a significant reduction not only of $\mathrm{HbA}_{1 c}$ levels but also of the frequency of hypoglycaemia, and also yielded high satisfaction rates.

Key words: clinical research, hypoglycaemia, insulin treatment, insulin glargine U300, type 1 diabetes.

\section{Introduction}

Insulin therapy is the mainstay treatment for type 1 diabetes mellitus (T1DM). Due to the pharmacological properties of exogenous insulin therapy, patients frequently develop undesirable conditions such as hypoglycaemia and hyperglycaemia [1]. In a metanalysis and review, it was stated that subcutaneous insulin infusion (SCII) therapy decreased se-

\author{
Corresponding author: \\ Savaş Volkan Kişioğlu \\ Health Sciences University \\ Kanuni Training and \\ Research Hospital \\ Trabzon, Turkey \\ E-mail: \\ volkankisioglu@yahoo.com
}


vere hypoglycaemia, while lowering haemoglobin glycated $\left(\mathrm{HbA}_{1 \mathrm{c}}\right)(-0.30 \%, 95 \% \mathrm{Cl}:-0.58$ to -0.02$)$ more than multiple dose injection (MDI) [2]; however, SCII therapy can be inaccessible for many patients due to its high costs. Moreover, some patients have difficulty adapting to SCII therapy and thus prefer MDI therapy [3].

Insulin glargine $100 \mathrm{U} / \mathrm{ml}$ (IGlar U100) is a basal human insulin analogue produced using recombinant DNA (rDNA) technology utilizing a non-pathogenic laboratory strain of Escherichia coli (E. coli) as the production organism. This analogue is produced by the addition of 2 arginines to the B30 position in human insulin and the substitution of asparagine for glycine at the A21 position. Although this modification allows IGlar U100 to remain soluble in its acidic $\mathrm{pH}$ in the injector, this analogue becomes less soluble at physiological $\mathrm{pH}$ upon transition to subcutaneous tissue, resulting in the formation of microprecipitates, from which small amounts of insulin glargine are slowly released [4, 5].

The effectiveness and safety of IGlar U100 have been shown in numerous studies [6-8]. Nevertheless, its risk of nocturnal hypoglycaemia increases when titrated to high doses to reach a target fasting blood glucose (FBG) level [6].

The above mentioned factors have led researchers to search for a new generation of insulin that has a longer duration of action, increased stability, and leads to less hypoglycaemia. Insulin glargine $300 \mathrm{U} / \mathrm{ml}$ (IGlar U300) is a highly concentrated form of insulin glargine that delivers $300 \mathrm{U}$ of insulin per $1 \mathrm{ml}$ of solution (one-third of the volume) and has a similar metabolism to that of IGlar U100 [9]. Moreover, IGlar U300 provides more steady-state pharmacokinetics (PK) and pharmacodynamics (PD) profiles and a longer duration of action than IGlar U100, extending blood glucose control well beyond $24 \mathrm{~h}$ [10]. On the other hand, IGlar U300 has been shown to be as effective for glycaemic control as IGlar U100 in patients with long-term T1DM and to have a lower risk of hypoglycaemia and weight gain than IGlar U100 [11, 12]. In a retrospective trial (SPARTA), IGlar U300 reduced the incidence of hypoglycaemic events while providing better blood glucose control [13]. These findings could not be demonstrated in EDITION 4, a randomized controlled trial [14].

In this study, we aimed to see whether IGlar U300 can provide better blood glucose control while reducing hypoglycaemia in a more homogeneous population compared to previous studies.

\section{Material and methods}

This retrospective study included patients who were diagnosed with T1DM at Karadeniz Technical University, Department of Endocrinology and Metabolism between Jan 2017 and May
2019. The study protocol was approved by Ethics Committee (approval date: 28 January 2020; no. 24237859-206). The study was conducted in accordance with the principles laid out by the $18^{\text {th }}$ World Medical Assembly (Helsinki, 1964) and all its subsequent amendments (up to 2013) and with the International Society for Pharmacoepidemiology guidelines for Good Pharmacoepidemiology Practice and local regulations, including local data protection regulations.

All forms of diabetes mediated by autoimmune $\beta$-cell destruction are included under the rubric of T1DM.

Inclusion criteria were as follows:

1) aged between $18-45$ years,

2) a diagnosis of T1DM established within a minimum of 12 months,

3) antibody positivity and C-peptide level $<1 \mathrm{mg} / \mathrm{dl}$,

4) history of daytime or nocturnal hypoglycemia during the diabetes treatment.

Exclusion criteria were as follows:

1) patients with acute coronary syndrome, acute cerebrovascular event, pregnancy, chronic liver disease, cancer and patients receiving dialysis due to end-stage renal disease,

2) use of drugs such as steroids that could elevate blood glucose level,

3) history of alcohol or drug abuse,

4) a previous of type 2 diabetes mellitus (T2DM),

5) non-compliant patients and those who did not take insulin regularly.

\section{Treatment planning}

Each patient was given a diabetic diet appropriate for their body mass index (BMI) by the dieticians in our hospital.

Target FBG level was defined as $80-130 \mathrm{mg} / \mathrm{dl}$, and the target postprandial glucose (PPG) level was defined as $<180 \mathrm{mg} / \mathrm{dl}$. The insulin regimen and doses administered by the patients throughout their treatment were recorded.

When switching from basal insulin regimen to IGlar U300, in patients who were switched to IGlar U300 due to the presence of hypoglycaemia, the total insulin dose was reduced by $20 \%$ and $50 \%$ of the total insulin dose was distributed to IGlar U300, and the remaining 50\% was appropriately distributed to 3 main meals based on a nutritional status and previous blood glucose measurements of the patients.

In the morning, the dose of IGlar U300 was increased by $2-4 \mathrm{U}$ if the FBG was $>130 \mathrm{mg} / \mathrm{dl}$, and it was increased by 4-8 U if the FBG was $>180 \mathrm{mg} /$ dl. However, it was reduced by $2-4 U$ if the FBG was $<70 \mathrm{mg} / \mathrm{dl}$ and was reduced by $4-8 \mathrm{U}$ if the FBG was $<56 \mathrm{mg} / \mathrm{dl}$.

Following the detection of PPG > $180 \mathrm{mg} / \mathrm{dl}$, the meal after which hypoglycaemia occurred was 
determined, and then the insulin dose before that meal was increased by $2-4 \mathrm{U}$ after questioning the patient's diet compliance.

Following the detection of PPG $<70 \mathrm{mg} / \mathrm{dl}$, the meal after which hypoglycaemia occurred was determined, and then the insulin dose before that meal was decreased by $2-4 \mathrm{U}$ after questioning the patient's diet compliance.

Each patient was advised to perform 7-point self-monitoring of blood glucose (SMBG) and to visit the outpatient clinic for a period of 5-7 days until reaching target glucose levels.

\section{Data collection and recording}

Clinical data of the patients were retrieved from electronic records. For each patient, data on $\mathrm{FBG}, \mathrm{HbA}_{1 \mathrm{c}}$, and weight levels over 3-month periods were evaluated. For patients with more than 1 record of FBG level over a 3-month period, the average level was taken for the analysis. For evaluation of FBG, $\mathrm{HbA}_{1 \mathrm{c}}$ and weight levels at 6 months and 12 months, observation windows of 120-240 days (4-8 months) and 240-480 days (9-16 months) after U300 initiation, respectively, were permitted. Final measurement was defined as the patient's last admission to the outpatient clinic between 9 and 16 months. Basal and bolus insulin doses were evaluated for each patient. Hypoglycaemia was defined as blood glucose level $<70 \mathrm{mg} / \mathrm{dl}$, either symptomatic or asymptomatic, measured in hospital or at home. In order to measure the satisfaction of the patients with their new basal insulin, the question was asked whether they would like to quit IGlar U300 and return to the previous basal insulin. Patients were asked to answer "Yes = Satisfied", "No = Unsatisfied", or "No significant difference". Hospitalization of patients due to ketoacidosis, blood glucose regulation, or severe hypoglycaemia episodes was recorded in the 12-month period before and after IG U300 transition.

\section{Biochemical analysis}

Fasting blood samples of all patients were taken from the antecubital vein after an overnight fasting period of at least $8 \mathrm{~h}$. Biochemical parameters were studied from plasma samples. Plasma glucose levels were measured using the enzymatic reference method with hexokinase (Beckman Coulter AU5800), and plasma $\mathrm{HbA}_{1 c}$ levels were measured by high-performance liquid chromatography (HPLC) and mass spectroscopy method (Premier HB9210). Low-density lipoprotein (LDL) was measured using enzymatic colorimetric assay (Beckman Coulter AU5800), and plasma creatinine was assessed using the kinetic Jaffé method (Beckman Coulter AU5800). Urine protein level was measured by the protein error of indicator method (IQ 200/iChem velocity).

\section{Statistical analysis}

Data were analysed using SPSS 23.0 for Windows (IBM Corp. Released 2015, Version 23.0. Armonk, NY: IBM Corp.). Descriptives were expressed as frequencies ( $n$ ) and percentages (\%) for categorical variables and as mean, median, standard deviation (SD), minimum, and maximum for continuous variables. Normal distribution of data was assessed using the one-sample Kolmogorov Smirnov test. Independent continuous variables were compared using the Mann-Whitney $U$ test because they did not show a normal distribution. For dependent continuous variables, 2 variables were compared using Wilcoxon signed-rank test, and 3 or more variables were compared using the Friedman test because they did not show a normal distribution. Dependent categorical variables were compared using McNemar's test. A $p$-value of $<0.05$ was considered significant.

\section{Results}

A total of 47 people were screened; 4 patients were excluded because of age $>45$ years, and 8 patients were excluded due to the absence of $\mathrm{HbA}_{1 \mathrm{c}}$ measurement at the $12^{\text {th }}$ month (9-16 months). The study included 35 patients with a mean age of $24.1 \pm 6.6$ years. The patients comprised 20 (57.1\%) females and 15 (42.9\%) males. Mean BMI was $24 \pm 3.8 \mathrm{~kg} / \mathrm{m}^{2}$. Twelve (34.2\%) patients were overweight, and 2 (5.7\%) patients were obese. Mean follow-up time was 12 months. Participants had a mean baseline $\mathrm{HbA}_{1 \mathrm{c}}$ of $9 \%(74.9 \mathrm{mmol} / \mathrm{mol})$, weight of $66.3 \mathrm{~kg}$, and BMI of $24 \mathrm{~kg} / \mathrm{m}^{2}$. The mean time from diagnosis of diabetes to data collection was 10.5 years. Table I presents the demographic and clinical characteristics of the patients.

At baseline, $100 \%$ of participants were on a basal-bolus insulin regimen. The most common basal insulins were U100 (77.8\%) and insulin detemir (35\%); insulin aspart (65.7\%) and insulin lispro (22\%) were the most commonly used rapid-acting insulins (Table II).

Accordingly, all the patients (100\%) received a basal-bolus insulin regimen, IGlar U100 was the most common basal insulin, and insulin aspart was the most common bolus insulin (Table II). The mean (SD) total daily insulin dose (basal and prandial insulin combined) at baseline was 54.5 $\pm 21.8 \mathrm{U} /$ day; the ratio of mean basal and prandial insulin dose was 46 : 54 (basal insulin: 22 [10-50] U/day, prandial insulin: 26 [6-76] U/day) and at final total insulin was $55.1 \pm 23.6$ units/day; the combination of mean basal and prandial insulin dose was 52 : 48 (basal insulin: 28 [6-54] U/day, 
Table I. Baseline participant characteristics $(n=35)$

\begin{tabular}{|c|c|}
\hline Variable & Value \\
\hline Age [year] mean \pm standard deviation & $24.1 \pm 6.6$ \\
\hline \multicolumn{2}{|l|}{ Sex, $n(\%):$} \\
\hline Male & $15(42.9)$ \\
\hline Female & $20(57.1)$ \\
\hline \multicolumn{2}{|l|}{ Ethnicity, $n(\%)$ : } \\
\hline White & $35(100)$ \\
\hline Weight $[\mathrm{kg}]$ mean \pm standard deviation & $66.3 \pm 11.6$ \\
\hline $\begin{array}{l}\text { BMI }\left[\mathrm{kg} / \mathrm{m}^{2}\right] \text { mean } \pm \text { standard } \\
\text { deviation }\end{array}$ & $24.4 \pm 7.4$ \\
\hline $\begin{array}{l}\text { Duration of diabetes [years] } \\
\text { mean } \pm \text { standard deviation }\end{array}$ & $10.5 \pm 5.96$ \\
\hline \multicolumn{2}{|l|}{ Hypertension, $n$ (\%): } \\
\hline Yes & $1(2.9)$ \\
\hline No & $34(97.1)$ \\
\hline Macrovascular complications, $n(\%)$ & $1(2.9)$ \\
\hline \multicolumn{2}{|l|}{ Microvascular complications, $n(\%)$ : } \\
\hline Retinopathy & $5(14.3)$ \\
\hline Neuropathy & $4(11.4)$ \\
\hline Nephropathy & $2(5.7)$ \\
\hline $\begin{array}{l}\text { Fasting blood glucose }[\mathrm{mg} / \mathrm{dl}] \\
\text { mean } \pm \text { standard deviation }\end{array}$ & $191.2 \pm 114.3$ \\
\hline $\begin{array}{l}\mathrm{HbA}_{1 \mathrm{c}}(\%)[\mathrm{mmol} / \mathrm{mol}] \\
\text { mean } \pm \text { standard deviation }\end{array}$ & $\begin{aligned} 9 & \pm 2 \\
{[74.9} & \pm 21.9]\end{aligned}$ \\
\hline
\end{tabular}

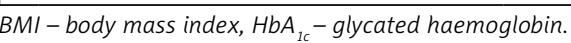

prandial insulin: 26 [5-64] U/day) (Table III). Diabetes-related microvascular complication documented at baseline, retinopathy (14.3\%) was the most common, followed by neuropathy (11.4\%).
Table II. Pre-study treatment regimens

\begin{tabular}{|lc|}
\hline Treatment & $\boldsymbol{N}(\%)$ \\
\hline Basal insulin + bolus insulin & $35(100)$ \\
\hline IGlar U100 & $28(80)$ \\
\hline Insulin Detemir & $7(20)$ \\
\hline Insulin Aspart & $23(65.7)$ \\
\hline Insulin Lispro & $8(22.2)$ \\
\hline Insulin Glulisine & $4(11.1)$ \\
\hline
\end{tabular}

\section{Efficacy}

\section{Change in $\mathrm{HbA}_{1 \mathrm{c}}$ and $\mathrm{FBG}$}

At month 6, FBG and $\mathrm{HbA}_{1 \mathrm{c}}$ levels were not decreased significantly when compared to baseline values ( $p=0.678$ and $p=0.199$, respectively). At the final visit of the patient, both $\mathrm{FBG}$ and $\mathrm{HbA}_{1 \mathrm{c}}$ levels were decreased significantly when compared to baseline values $(p<0.043$ and $p<0.025$, respectively).

\section{Change in weight}

When compared baseline, no significant difference was found in month 6 and final body weight ( $p=0.835$ and $p=0.796$, respectively).

\section{Hypoglycaemia}

Of the 35 patients, $100 \%$ were switched to IGlar U300 due to frequent hypoglycaemia. Before switching to IGlar U300, 7 (20\%) patients experienced hypoglycaemia every day of the week (very frequent), 20 (57.1\%) patients more

Table III. Clinical and laboratory parameters before and after the use of IGlar U300

\begin{tabular}{|c|c|c|c|}
\hline Parameter & $\begin{array}{l}\text { Baseline } \\
(n=35)\end{array}$ & $\begin{array}{c}\text { Final }^{\epsilon} \\
(n=35)\end{array}$ & $P$-value \\
\hline Systolic blood pressure (SBP) [mm Hg] & $120[90-150]$ & $120[90-150]$ & $0.676^{*}$ \\
\hline Diastolic blood pressure (DBP) [mm Hg] & $70[60-90]$ & $70[50-90]$ & $0.394^{*}$ \\
\hline Total dose of basal insulin [U] & $22[10-50]$ & $28[6-54]$ & $0.158^{*}$ \\
\hline IGlar U100 - IGlar U300 dose [U] & $26.1 \pm 9.6$ & $27.9 \pm 9.7$ & $0.203^{* *}$ \\
\hline I Detemir dose - IGlar U300 dose [U] & $16[10-50]$ & $14[8-54]$ & $1.000^{*}$ \\
\hline IGlar U300 dose [U] & $24[8-50]$ & $28[6-54]$ & $0.144^{*}$ \\
\hline Total dose of bolus insulin [U] & $26[6-76]$ & $26[5-64]$ & $0.255^{*}$ \\
\hline Ratio of total dose of injected insulin to weight [unit/kg/day] & $0.8 \pm 0.3$ & $0.8 \pm 0.3$ & $0.713^{* *}$ \\
\hline Alanine aminotransferase (ALT) (N: 0-45 U/l) & $12[3-56]$ & $10[3-100]$ & $0.910^{*}$ \\
\hline Low-density lipoprotein $(\mathrm{N}:<160 \mathrm{mg} / \mathrm{dl})$ & $99[44-190]$ & $99.5[42-190]$ & $0.483^{*}$ \\
\hline Creatinine ( $\mathrm{N}: 0.67-1.17 \mathrm{mg} / \mathrm{dl}$ ) & $0.6[0.4-1.6]$ & $0.6[0.4-1.3]$ & $0.680^{*}$ \\
\hline Glomerular filtration rate (GFR) [ml/min] & $130[54-148]$ & $131[69-152]$ & $0.210^{*}$ \\
\hline Proteinuria ( $\mathrm{N}:<50 \mathrm{mg} / \mathrm{dl})$ & $0[0-100]$ & $0[0-50]$ & $0.907^{\star}$ \\
\hline Hospitalization $(n)$ & 14 & 9 & \\
\hline Ketosis & 3 & 1 & \\
\hline
\end{tabular}

${ }^{*}$ Wilcoxon signed-rank test, ${ }^{* *}$ Paired t-test. ${ }^{\epsilon}$ Final measurement was defined as the patient's last admission to the outpatient clinic between 9 and 16 months. 
Savaş Volkan Kişioğlu, Ahmet Suat Demir, Damla Tufekci, Yasemin Emur Gunay, Hulya Coskun, Ozge Ucuncu, Irfan Nuhoglu, Mustafa Kocak, Serdar Karakullukcu, Halil Onder Ersoz

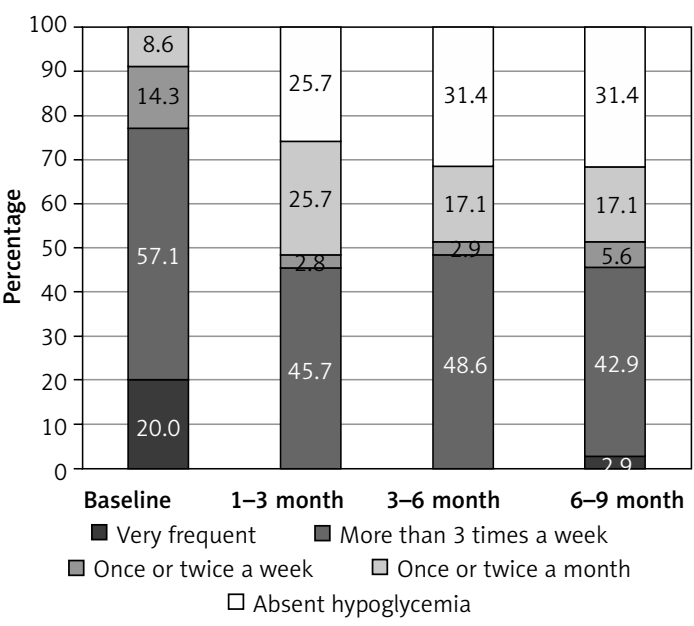

Figure 1. Hypoglycemia prevalence distribution after transition to IGlar U300

than 3 times a week, $5(14.3 \%)$ patients once or twice a week, and 3 (8.6\%) patients once or twice a month. After switching to the IGlar U300, between 3 and 6 months, 17 (48.6\%) patients experienced hypoglycaemia more than 3 times a week, 1 (2.9) patient once or twice a week, and 6 (17.1) patients once or twice a month (Figure 1). While there were no patients with frequent hypoglycaemia, 11 (31.4) patients no longer experienced hypoglycaemia.

\section{Ketosis and hospitalization}

Fourteen patients (11 patients due to blood glucose regulation or severe episodes of hypoglycaemia) were hospitalized in the 12-month period before IGlar U300 transition and 9 patients (8 patients due to blood glucose regulation or severe episodes of hypoglycaemia) in the 12-month period after transition.

\section{Satisfaction}

Of the 26 patients who declared their satisfaction, 23 (88.5\%) were satisfied, 2 (7.7\%) indicated that there was no significant difference, and $1(3.8 \%)$ patient was unsatisfied (Figure 2 ).

\section{Discussion}

The patients included in the study were fed up with treatment and in constant fear of hypoglycaemia due to frequent episodes, and they constituted a difficult patient group whose blood glucose control could not be controlled. These patients were initiated on IGlar U300 with high hopes, and it was explained to them that the treatment could lead to a significant reduction in the frequency of hypoglycaemia. There were few cases of documented, severe blood glucose regulation problems, severe episodes of hypoglycaemia, and dia-

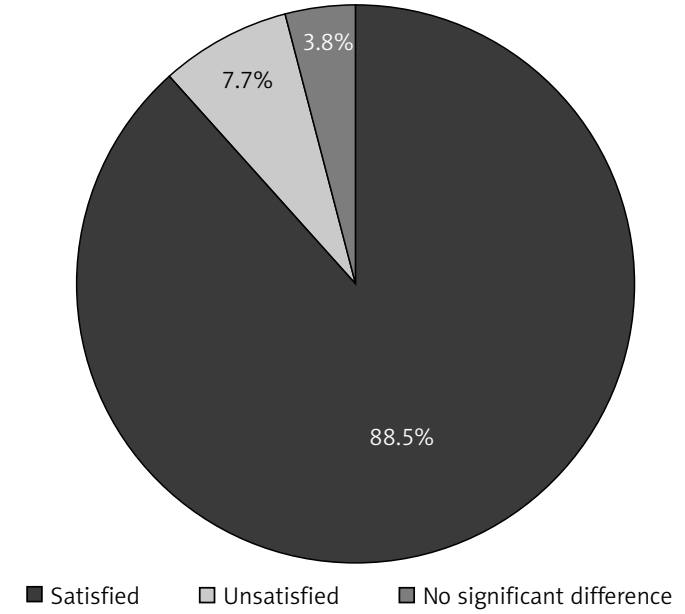

Figure 2. Satisfaction rates regarding the use of IGlar U300

betic ketoacidosis requiring hospitalization before and after U300 treatment was initiated.

The absence of a control group in our study does not reduce the importance of our study. Due to the retrospective nature of the study, routine polyclinic operation continued after the patients were switched to IGlar U300 for blood glucose regulation, no privileges were provided to the patients, and the necessity of appointment for follow-up continued. For this reason, $\mathrm{HbA}_{1 \mathrm{c}}$ values of all patients could not be examined periodically (in the $9^{\text {th }}$ month, $12^{\text {th }}$ month, $15^{\text {th }}$ month) in the follow-up period, and the values examined in a wide time interval, such as 9 to 16 months, were taken as the final values of the patients. However, because they did not perform periodic visits, we questioned them about whether they had taken insulin regularly and followed their diet, and we recorded their responses at each visit.

Although the final IGlar U300 dosage was higher than the baseline basal insulin dosage in all patients, no significant difference was established $(p=0.144)$. Similarly, no significant difference was found between baseline and final total bolus insulin dosages $(p=0.255)$ and between baseline and the final ratio of the total dose of injected insulin to weight $(\mathrm{U} / \mathrm{kg} / \mathrm{day})(p=0.713)$. Taken together, these findings indicate that although a minimal increase occurred in the insulin dosages of the patients, a reduction occurred in the frequency of hypoglycaemia. Previous randomized open-label studies reported that although switching to IGlar U300 led to a significant reduction in the frequency of hypoglycaemia, it provided suboptimal blood glucose control $[11,12]$. In the Edition 4 study, which included 274 patients randomized to Gla300 and 275 to Gla-100, and a patient follow-up of 6 months, the change in $\mathrm{HbA}_{1 c}$ was equivalent in the 2 treatment groups (difference $0.04 \%$, $95 \% \mathrm{Cl}:-0.10$ to 0.19$)$ ( $0.4 \mathrm{mmol} / \mathrm{mol},-1.1$ to 2.1 ), 
and Gla-300 was thus deemed noninferior. In the SPARTA study, 298 T1DM patients over a 6-month period, in a similar way to our study, reported that the $\mathrm{HbA}_{1 \mathrm{c}}$ levels decreased significantly $(-4 \mathrm{mmol} /$ mol, $-0.4 \% ; p<0.001 ; n=188$ ) and the incidence of reported hypoglycaemia decreased, although no significant difference was noted in the insulin doses [13]. In our study, an evaluation of the FBG and $\mathrm{HbA}_{1 \mathrm{c}}$ values in 35 patients followed up over a 9-16-month period. showed a significant reduction in final FBG and $\mathrm{HbA}_{1 c}$ values $(p=0.043$ and $p=0.025$, respectively).

When our study, the SPARTA study, and Edition 4 are compared, the difference in study populations draws attention: patient ages were, respectively, 24.4 (7.4) years vs. 42.1 (14) years vs. 46.4 (13.9), duration of diabetes was 10 (6) years vs. 20.3 (12.9) years vs. 20.5 (12.7) years, and BKI was $24(3.8) \mathrm{kg} / \mathrm{m}^{2}$ vs. $28.3(6.7) \mathrm{kg} / \mathrm{m}^{2}$ vs. $27.6(5.5) \mathrm{kg} / \mathrm{m}^{2}$. In our study, unlike the other 2 studies, younger and lower weight patients with short-term diabetes were included. Because patients under the age of 45 years were included in our study, it was observed that the patients were more homogeneously distributed. In the SPARTA study, the most common reason for switching to IGlar U300 was the low efficacy of previous basal insulin (157/298 (53\%)). In addition, in the SPARTA study, before the transition of IGlar U300, $55 \%$ of the patients were using IGlar U100. In our study and the Edition 4 study, respectively, $80 \%$ and $85 \%$ IGlar U100 were used before switching to U300. In our study, unlike the SPARTA study, in the 6th month no significant decrease was found in FBG and $\mathrm{HbA}_{1 \mathrm{c}}$ (Table IV). In the SPARTA study, it could be that there was a significant decrease in FPG and $\mathrm{HbA}_{1 \mathrm{c}}$ in the majority of patients includ- ed in the study due to the ineffectiveness of old basal insulin, and the use of basal insulins other than IGlar U100 was relatively high. In the SPARTA study, $19 \%$ of patients switched to IGlar U300 due to the risk of hypoglycaemia. Although all the patients included in our study have a risk of hypoglycaemia, administration of IGlar U300 provided better blood glucose regulation over 9-16 months period. Although there was a numerical decrease in $\mathrm{FBG}$ and $\mathrm{HbA}_{1 \mathrm{c}}$ in the 6th month, it is interesting that this decrease, which was not statistically significant, became meaningful in the follow-up period. This could be attributed to the possibility that patients might have reduced or skipped their insulin doses due to the fear of hypoglycaemia after noticing their relatively low blood glucose levels, which were measured before switching to IGlar U300 (70-100 mg/dl) and the reduction in the frequency of hypoglycemia in patients may have enabled us to set a lower blood glucose target while titrating basal insulin.

A total of 26 patients were asked if they were satisfied with their new basal insulin or would like to return to their old basal insulins, and the majority of them (88.5\%) indicated that they were satisfied with IGlar U300 and did not want to return to basal insulins. This finding alone is highly important due to the fact that living without fear of hypoglycaemia will lead to a significant improvement in patients' quality of life.

Although there are several randomized studies indicating a reduction in body weight, this effect remains unelucidated, and it could be associated with the lower peak-to-trough ratio of IGlar U300 [11]. In our study, no significant difference was found between baseline and 6-month and final body weight values, which could be attributed to

Table IV. Comparison of baseline and final body weight, $\mathrm{FBG}$, and $\mathrm{HbA}_{1 \mathrm{c}}$ values $(n=35)$

\begin{tabular}{|c|c|c|c|c|}
\hline \multirow[t]{2}{*}{ Parameter } & \multirow[t]{2}{*}{$N$} & & & \\
\hline & & \multicolumn{2}{|c|}{ Means } & $P$-value** \\
\hline \multicolumn{5}{|c|}{ Fasting blood glucose $[\mathrm{mg} / \mathrm{dl}]$ mean \pm SD: } \\
\hline Baseline - 6 month $^{*}$ & 35 & $191.2 \pm 114.3$ & $180.8 \pm 97.9$ & 0.678 \\
\hline Baseline - Final ${ }^{€}$ & 35 & $191.2 \pm 114.3$ & $149.5 .3 \pm 62.4$ & 0.043 \\
\hline 6 month - Final f $^{-}$ & 35 & $180.8 \pm 97.9$ & $149.5 .3 \pm 62.4$ & 0.054 \\
\hline \multicolumn{5}{|c|}{$\mathrm{HbA}_{1 \mathrm{c}}(\%)[\mathrm{mmol} / \mathrm{mol}]$ mean $\pm \mathrm{SD}:$} \\
\hline Baseline - 6 month $^{*}$ & 35 & $9 \pm 2[74.9 \pm 21.9]$ & $8.9 \pm 2[73.8 \pm 21.9]$ & 0.199 \\
\hline Baseline - Final $\left.\right|^{€}$ & 35 & $9 \pm 2[74.9 \pm 21.9]$ & $8.7 \pm 1.6[71.6 \pm 17.5]$ & 0.025 \\
\hline 6 month - Final ${ }^{€}$ & 35 & $8.9 \pm 2[73.8 \pm 21.9]$ & $8.7 \pm 1.6[71.6 \pm 17.5]$ & 0.179 \\
\hline \multicolumn{5}{|l|}{ Weight $[\mathrm{kg}]$ mean $\pm \mathrm{SD}$} \\
\hline Baseline - 6 month $^{*}$ & 35 & $66.3 \pm 11.6$ & $66.4 \pm 12.1$ & 0.835 \\
\hline Baseline - Final€ & 35 & $66.3 \pm 11.6$ & $66.5 \pm 12.8$ & 0.796 \\
\hline 6 month - Final ${ }^{€}$ & 35 & $66.4 \pm 12.1$ & $66.5 \pm 12.8$ & 0.811 \\
\hline
\end{tabular}

**Paired t-test. ${ }^{*} 6$-month measurement was defined as the patient's admission to the outpatient clinic between 4 and 8 months. ${ }^{\epsilon}$ Final measurement was defined as the patient's last admission to the outpatient clinic between 9 and 16 months. 
the use of similar numbers of insulin units and to the use of IGlar U100 as the basal insulin, which has similar molecular characteristics to those of IGlar U300.

Our study was limited in several ways. First, it had a retrospective design. Second, not all the patients completed the 18-month follow-up period, and thus the measurements obtained during their last clinical visit were accepted as final measurements. Finally, the frequency of hypoglycaemia was calculated based on the episodes of hypoglycaemia reported by the patients and was not calculated by using SCII, the high costs of which are not covered by insurance programs.

In conclusion, the present study was conducted among a challenging patient group of 35 T1DM patients who received IGlar U300. In this challenging patient group, IGlar U300 led to a significant reduction not only of $\mathrm{HbA}_{1 \mathrm{c}}$ levels but also of the frequency of hypoglycaemia, and it also yielded high satisfaction rates.

\section{Conflict of interest}

The authors declare no conflict of interest.

\section{References}

1. Standards of Medical Care in Diabetes -2020 . Diabetes Care 2020; 43 (Suppl 1): S1-2.

2. Yeh HC, Brown TT, Maruthur N, et al. Comparative effectiveness and safety of methods of insulin delivery and glucose monitoring for diabetes mellitus: a systematic review and meta-analysis. Ann Intern Med 2012; 157: 336-47.

3. Schifferdecker E, Schmidt K, Boehm BO, et al. Long-term compliance of intensified insulin therapy. Diabetes Res Clin Practice 1994; 23: 17-23.

4. Wang F, Carabino JM, Vergara CM. Insulin glargine: a systematic review of a long-acting insulin analogue. Clin Therapy 2003; 25: 1541-77.

5. Bolli GB, DeVries JH. New long-acting insulin analogs: from clamp studies to clinical practice. Diabetes Care 2015; 38: 541-3.

6. Riddle MC, Rosenstock J, Gerich J. The treat-to-target trial: randomized addition of glargine or human NPH insulin to oral therapy of type 2 diabetic patients. Diabetes Care 2003; 26: 3080-6.

7. Gerstein HC, Bosch J, Dagenais GR, et al. Basal insulin and cardiovascular and other outcomes in dysglycemia. N Engl J Med 2012; 367: 319-28.

8. Davies M, Lavalle-González F, Storms F, et al. Initiation of insulin glargine therapy in type 2 diabetes subjects suboptimally controlled on oral antidiabetic agents: results from the ATLANTUS trial. Diabetes Obes Metabolism 2008; 10: 387-99.

9. Steinstraesser A, Schmidt R, Bergmann K, et al. Investigational new insulin glargine $300 \mathrm{U} / \mathrm{ml}$ has the same metabolism as insulin glargine $100 \mathrm{U} / \mathrm{ml}$. Diabetes Obes Metabol 2014; 16: 873-6.

10. Becker RH, Dahmen R, Bergmann K, et al. New insulin glargine 300 units $\mathrm{mL}-1$ provides a more even activity profile and prolonged glycemic control at steady state compared with insulin glargine 100 units $\mathrm{mL}-1$. Diabetes Care 2015; 38: 637-43.

11. Home PD, Bergenstal RM, Bolli GB, et al. New insulin glargine 300 Units $/ \mathrm{Ml}$ versus glargine 100 units $/ \mathrm{mL}$ in people with type 1 diabetes: a randomized, phase $3 a$, open-label clinical trial (EDITION 4). Diabetes Care 2015; 38: 2217-25.

12. Danne T, Matsuhisa M, Sussebach C, et al. Lower risk of severe hypoglycaemia with insulin glargine $300 \mathrm{U} / \mathrm{mL}$ versus glargine $100 \mathrm{U} / \mathrm{mL}$ in participants with type 1 diabetes: a meta-analysis of 6-month phase 3 clinical trials. Diabetes Obes Metabol 2020; 22: 1880-5.

13. Pang T, Bain SC, Neil R, et al. A multicentre, UK, retrospective, observational study to assess the effectiveness of insulin glargine 300 units $/ \mathrm{ml}$ in treating people with type 1 diabetes mellitus in routine clinical practice (SPARTA). Diabetic Med 2019; 36: 110-9.

14. Bergenstal RM, Bailey TS, Rodbard D, et al. Comparison of insulin glargine $300 \mathrm{U} / \mathrm{mL}$ and $100 \mathrm{U} / \mathrm{mL}$ in adults with type 1 diabetes: continuous glucose monitoring profiles and variability using morning or evening injections. Diabetes Care 2017; 40: 554-60. 